\title{
Endoscopic Full-Thickness Resection Combined with Laparoscopic Surgery
}

\author{
Chan Gyoo Kim \\ Center for Gastric Cancer, National Cancer Center, Goyang, Korea
}

Endoscopic full-thickness resection combined with laparoscopic surgery was recently developed. These procedures could be categorized as "Cut first and then suture" and "Suture first and then cut". "Cut first and then suture" includes laparoscopic and endoscopic cooperative surgery (LECS) and laparoscopy-assisted endoscopic full-thickness resection (LAEFR). Recent studies have demonstrated the safety and efficacy of LECS and LAEFR. However, these techniques are limited by the related exposure of the tumor and gastric mucosa to the peritoneal cavity and manipulation of these organs, which could lead to viable cancer cell seeding and the spillage of gastric juice into the peritoneal cavity. In the "Suture first and then cut" technique, the serosal side of the stomach is sutured to invert the stomach and subsequently endoscopic resection is performed. In this article, details of these techniques, including their advantages and limitations, are described. Clin Endosc 2018;51:33-36

Key Words: Endoscopic full-thickness resection; Laparoscopic and endoscopic cooperation surgery; Non-exposed endoscopic wallinversion surgery; Non-exposure simple suturing endoscopic full-thickness resection

\section{INTRODUCTION}

Intraoperative endoscopy (performing endoscopy during laparoscopic or open surgery) is most commonly used to identify tumor margins or detect anastomosis site bleeding. Intraoperative endoscopic resection is also under development. As minimal tissue resection is the main purpose of intraoperative endoscopic resection, it is usually performed with laparoscopic surgery rather than with open surgery. Endoscopic submucosal dissection (ESD) for early gastric cancer (EGC) generally does not require a laparoscopic

Received: September 21, 2017 Revised: November 29, 2017

Accepted: November 30, 2017

Correspondence: Chan Gyoo Kim

Center for Gastric Cancer, National Cancer Center, 323 Ilsan-ro, Ilsandong-gu, Goyang 10408, Korea

Tel: +82-31-920-1620, Fax: +82-31-920-2798, E-mail: glse@ncc.re.kr

(cc) This is an Open Access article distributed under the terms of the Creative Commons Attribution Non-Commercial License (http://creativecommons.org/ licenses/by-nc/3.0) which permits unrestricted non-commercial use, distribution, and reproduction in any medium, provided the original work is properly cited. procedure. Endoscopic full-thickness resection (EFTR) for small tumors can also be performed without laparoscopy. ${ }^{1-3}$ However, EFTR for most tumors usually require laparoscopy, which enables suturing of resection sites and control of bleeding from the outer bowel wall. ${ }^{4}$ Combining the EFTR and laparoscopic approaches could be called hybrid natural orifice transluminal endoscopic surgery (NOTES). NOTES is a minimally invasive surgery that accesses the peritoneal or thoracic spaces through the natural orifice, such as the mouth or anus. Owing to the technical limitations of the standard NOTES technique, the NOTES technique can combine a transcutaneous rigid laparoscopic approach, which is called hybrid NOTES technique. ${ }^{4}$

The EFTR techniques with laparoscopic surgery include laparoscopic and endoscopic cooperative surgery (LECS), laparoscopy-assisted endoscopic full-thickness resection (LAEFR), non-exposed endoscopic wall-inversion surgery (NEWS), and non-exposure simple suturing endoscopic full-thickness resection (NESS-EFTR). These techniques were categorized as "Cut first and then suture" and "Suture first 
and then cut". Here, the advantages and limitations of these techniques are described.

\section{CUT FIRST AND THEN SUTURE}

Hiki et al. first reported the use of LECS in $2008 .{ }^{5}$ In this procedure, the endoscopist makes the circumferential submucosal incision and then perforates the stomach wall using a needle knife. Seromuscular dissection and closure of the opening are performed laparoscopically at the peritoneum. Before the endoscopic resection, blood vessels on the expected excision area are removed using a laparoscopic ultrasonically activated device. As a first step of the endoscopic intervention, the lateral margin of the tumor is marked using argon plasma coagulation. Endoscopic circumferential mucosal incision outside of the marked area is performed as in the ESD technique. An artificial perforation is created using a needle knife, along the opening of the submucosal layer (incision line of the circumferential mucosal incision). Thereafter, the laparoscopic procedure is performed. The tip of the ultrasonically activated device is inserted in the perforation at the start of the seromuscular dissection. After two- to three-fourth of the circumference of the tumor is resected, the tumor is turned over toward the peritoneal cavity. The tumor is lifted up by the laparoscopic accessory, and the tumor is resected and sutured using a laparoscopic stapler. ${ }^{5}$

The benefit of LECS over conventional laparoscopic wedge resection is the direct endoscopic intraluminal visualization of the tumor during the resection. Laparoscopic wedge resection for extraluminally growing tumors is generally considered an easy procedure. However, excessive resection of gastric tissue may occur during the laparoscopic resection of intraluminal growing tumors. Excessive gastric resection may lead to postoperative deformity of the stomach with consequent gastric stasis. Direct endoscopic visualization and peritumoral circumferential incision could lead to minimal resection of the gastric tissue.

Abe et al. also reported a similar procedure called LAEFR in 2008 , in which the role of endoscopy was increased. ${ }^{6}$ Endoscopic submucosal incision around the circumference of the tumor following endoscopic wall perforation is the same in LECS and LAEFR. Thereafter, an endoscopic full-thickness cut around three-fourths of the circumference of the submucosal incision line is performed. Finally, seromuscular incision and suturing were performed laparoscopically.

The LECS and LAEFR results are summarized in Table 1 . $^{5-13}$ The largest recent retrospective study showed that LECS is safe and effective. ${ }^{13}$ This retrospective study included $100 \mathrm{pa}-$ tients who underwent LECS for subepithelial tumors (SETs).
Complete resection with negative surgical margins was achieved in all cases, including nine tumors at the gastric cardia. The mean operation time was 174 minutes, with minimal estimated blood loss $(16 \mathrm{~mL})$. The mean time until the initiation of oral intake was 1.4 days. The only severe adverse event was leakage, which occurred in one patient. No case of local or distant tumor recurrence was observed. In Japan, this technique is approved and covered by health insurance. ${ }^{13}$

Thus far, LECS and LAEFR studies have primarily focused on resection of SET. Reports of treating EGC with LECS or LAEFR are uncommon because of the risk of lymph node metastasis. Cho et al. reported 14 cases of EGC treated with LAEFR after sentinel lymph node navigation. ${ }^{8}$ All cases were resected en bloc with negative resection margins, but five required conversion to subtotal gastrectomy. The reasons of conversion to gastrectomy were reconstruction problems (twisted or hourglass shape), insufficient vascular flow after anastomosis, and leakage. The median tumor size was $26 \mathrm{~mm}$ (range, 12-90 mm), and the median operating time was 143 minutes (range, 110-253 minutes). The estimated blood loss volume of the successful cases was $16 \mathrm{~mL}$ (range, 5-30 mL). Currently, a randomized controlled prospective trial to evaluate the sentinel lymph node navigation for EGC (SENORITA trial) is ongoing. ${ }^{14}$ Further EFTR studies to evaluate the usefulness of EFTR for EGC will be performed if the SENORITA trial proves the safety and effectiveness of sentinel lymph node navigation for EGC.

\section{SUTURE FIRST AND THEN CUT}

Surgical procedures, as well as LECS and LAEFR, generally involve resection, followed by suturing. The limitation of the "Cut first and then suture" method is that the tumor and gastric mucosa are exposed to and manipulated within the peritoneal cavity. It is hard to completely avoid viable tumor cell seeding into the peritoneal cavity. Therefore, SET without an ulcer was considered an indication for LECS. ${ }^{5}$ Possible infection or inflammation caused by the spillage of gastric juices into the abdominal cavity is another concern. "Suture first and then cut", techniques were developed to overcome this limitation. "Suture first and then cut" means that the stomach wall is first laparoscopically sutured from the peritoneal cavity, resulting in inversion of the stomach wall. Endoscopic resection is then performed. The tumor tissue is retrieved via the oral cavity, meaning that the tumor is not exposed to the peritoneum (non-exposure technique).

NEWS is the first "Suture first and then cut" technique. ${ }^{15}$ In NEWS, a circumferential seromuscular incision is performed laparoscopically after markings are placed on the mucosal and 


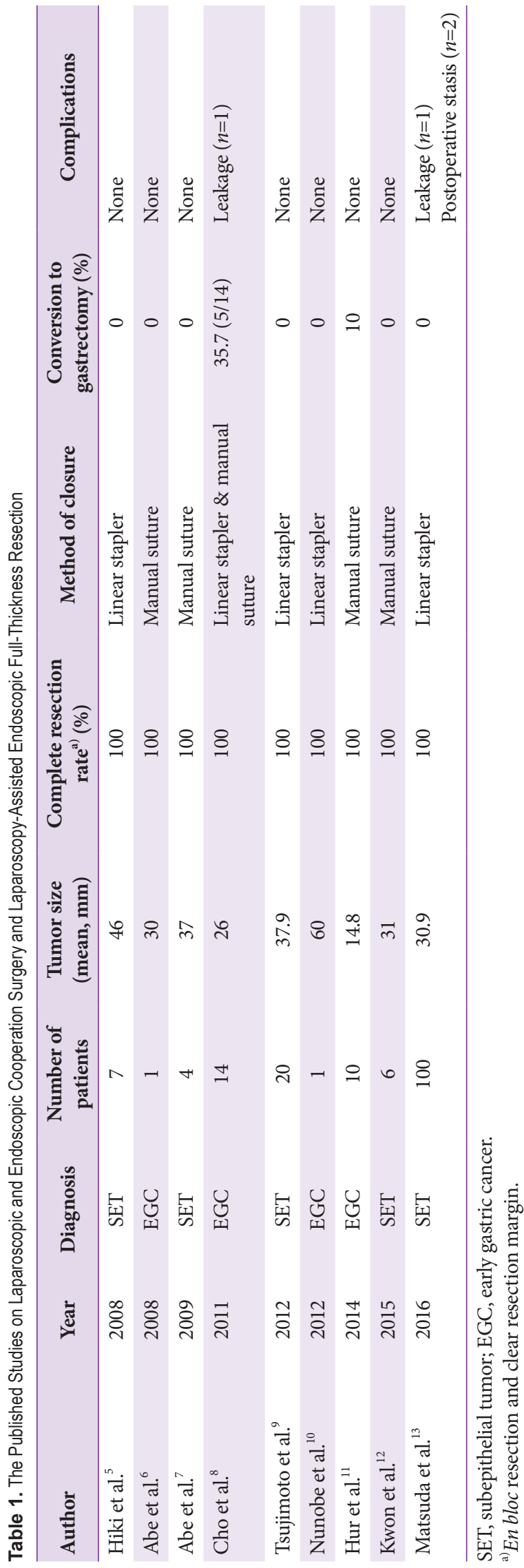

serosal sides, and a circumferential submucosal fluid cushion is created endoscopically. Subsequently, the seromuscular layers are linearly sutured with the lesion inverted to the intraluminal side, and a surgical sponge is placed in the space between the suturing and serosal planes of the inverted lesion. The lesion is finally resected endoscopically. ${ }^{16,17}$ The initial report with six SET patients and a following report with 20 SET patients showed successful outcomes. En bloc full-thickness resection with clear margins was possible in all patients. No severe intraoperative or postoperative adverse events developed. Perforations developed in 33\% (2/6) of the patients in the initial study, but this rate improved to $5 \%$ in the later report. ${ }^{15,17}$ The limitation of this technique is that it requires difficult procedures, such as laparoscopic circumferential seromuscular incisions, surgical sponge insertion, and laparoscopic muscular suturing along the incision sites. Laparoscopic intracorporeal suturing and knot tying for anastomoses are considered the most difficult and time-consuming tasks in laparoscopic surgery because of the confined space and limited visualization. ${ }^{18}$

Kim et al. proposed a similar NESS technique that skips the laparoscopic circumferential seromuscular incisions (NESS-EFTR). ${ }^{19}$ Instead of using a circumferential seromuscular incision followed by suturing along the incision line and knot tying, the serosal side (seromuscular layer) is sutured using a barbed suture thread; the mucosal side, with an endoloop and clips. The barbed suture thread eliminates the need to tie a knot, saves procedure time, and simplifies the procedure. An endoscopic mucosal suture was added to provide a secure anastomosis. Until now, only animal studies have been published, but clinical trials (NCT02764944 and NCT03216174) are ongoing.

Combination of laparoscopic and endoscopic approaches for neoplasia with a non-exposure technique (CLEAN-NET) was proposed as a non-exposed technique. ${ }^{20}$ In the CLEANNET, endoscopy is used to identify the tumor margin, but endoscopic resection is not performed. Resection and suturing are performed laparoscopically. Thus, its details are not discussed in this article.

\section{CONCLUSIONS}

The initial role of endoscopy in endoscopic resection combined with laparoscopic surgery was endoscopic perforation. Subsequent studies have enabled EFTR and endoscopic suturing, which is combined by laparoscopy. The role of endoscopy will be increased to achieve minimal invasive surgery. 


\section{Conflicts of Interest}

The Author has no financial conflicts of interest.

\section{Acknowledgments}

This work was supported by grant No. 1710280 from the National Cancer Center, Korea.

\section{REFERENCES}

1. Schlag C, Wilhelm D, von Delius S, Feussner H, Meining A. EndoResect study: endoscopic full-thickness resection of gastric subepithelial tumors. Endoscopy 2013;45:4-11.

2. Shi Q, Chen T, Zhong YS, et al. Complete closure of large gastric defects after endoscopic full-thickness resection, using endoloop and metallic clip interrupted suture. Endoscopy 2013;45:329-334.

3. Zhou PH, Yao LQ, Qin XY, et al. Endoscopic full-thickness resection without laparoscopic assistance for gastric submucosal tumors originated from the muscularis propria. Surg Endosc 2011;25:2926-2931.

4. Kim CG. Natural orifice transluminal endoscopic surgery and upper gastrointestinal tract. J Gastric Cancer 2013;13:199-206.

5. Hiki N, Yamamoto Y, Fukunaga T, et al. Laparoscopic and endoscopic cooperative surgery for gastrointestinal stromal tumor dissection. Surg Endosc 2008;22:1729-1735.

6. Abe N, Mori T, Takeuchi H, et al. Successful treatment of early stage gastric cancer by laparoscopy-assisted endoscopic full-thickness resection with lymphadenectomy. Gastrointest Endosc 2008;68:1220-1224.

7. Abe N, Takeuchi H, Yanagida O, et al. Endoscopic full-thickness resection with laparoscopic assistance as hybrid NOTES for gastric submucosal tumor. Surg Endosc 2009;23:1908-1913.

8. Cho WY, Kim YJ, Cho JY, et al. Hybrid natural orifice transluminal endoscopic surgery: endoscopic full-thickness resection of early gastric cancer and laparoscopic regional lymph node dissection--14 human cases. Endoscopy 2011;43:134-139.

9. Tsujimoto H, Yaguchi Y, Kumano I, Takahata R, Ono S, Hase K. Successful gastric submucosal tumor resection using laparoscopic and endoscopic cooperative surgery. World J Surg 2012;36:327-330.
10. Nunobe S, Hiki N, Gotoda T, et al. Successful application of laparoscopic and endoscopic cooperative surgery (LECS) for a lateral-spreading mucosal gastric cancer. Gastric Cancer 2012;15:338-342.

11. Hur H, Lim SG, Byun C, et al. Laparoscopy-assisted endoscopic full-thickness resection with basin lymphadenectomy based on sentinel lymph nodes for early gastric cancer. J Am Coll Surg 2014;219:e29-e37.

12. Kwon OK, Yu W. Endoscopic and laparoscopic full-thickness resection of endophytic gastric submucosal tumors very close to the esophagogastric junction. J Gastric Cancer 2015;15:278-285.

13. Matsuda T, Hiki N, Nunobe S, et al. Feasibility of laparoscopic and endoscopic cooperative surgery for gastric submucosal tumors (with video). Gastrointest Endosc 2016;84:47-52.

14. Park JY, Kim YW, Ryu KW, et al. Assessment of laparoscopic stomach preserving surgery with sentinel basin dissection versus standard gastrectomy with lymphadenectomy in early gastric cancer-a multicenter randomized phase III clinical trial (SENORITA trial) protocol. BMC Cancer 2016;16:340.

15. Mitsui T, Niimi K, Yamashita H, et al. Non-exposed endoscopic wall-inversion surgery as a novel partial gastrectomy technique. Gastric Cancer 2014;17:594-599.

16. Goto O, Mitsui T, Fujishiro M, et al. New method of endoscopic full-thickness resection: a pilot study of non-exposed endoscopic wall-inversion surgery in an ex vivo porcine model. Gastric Cancer 2011;14:183-187.

17. Goto O, Takeuchi H, Sasaki M, et al. Laparoscopy-assisted endoscopic full-thickness resection of gastric subepithelial tumors using a nonexposure technique. Endoscopy 2016;48:1010-1015.

18. Milone M, Di Minno MN, Galloro G, et al. Safety and efficacy of barbed suture for gastrointestinal suture: a prospective and randomized study on obese patients undergoing gastric bypass. J Laparoendosc Adv Surg Tech A 2013;23:756-759.

19. Kim CG, Yoon HM, Lee JY, et al. Nonexposure endolaparoscopic full-thickness resection with simple suturing technique. Endoscopy 2015;47:1171-1174

20. Inoue $\mathrm{H}$, Ikeda $\mathrm{H}$, Hosoya $\mathrm{T}$, et al. Endoscopic mucosal resection, endoscopic submucosal dissection, and beyond: full-layer resection for gastric cancer with nonexposure technique (CLEAN-NET). Surg Oncol Clin N Am 2012;21:129-140. 